\title{
Microlens array-based exit pupil expander for full color display applications
}

\author{
Hakan Urey ${ }^{\mathrm{a}}$, Karlton D. Powell ${ }^{\mathrm{b}}$ \\ ${ }^{a}$ Optical Microsystems Laboratory and Department of Electrical Engineering \\ Koç University, Sariyer, Istanbul, TURKEY \\ ${ }^{\mathrm{b}}$ Microvision Inc., 19910 North Creek Pkwy, Bothell, WA 98011, USA \\ E-mails: hurey@ku.edu.tr, karlton_powell@microvision.com
}

\begin{abstract}
Retinal Scanning Display (RSD) is a near-to-eye scanned beam display technology. An exit pupil expander (EPE) or numerical aperture (NA) expander is used in RSDs to create a large display exit pupil. A novel EPE approach that uses two microlens arrays (MLA) is presented in this paper. The approach is based on cascading two identical microlens arrays spaced precisely at one focal length distance with sub-micron registration tolerances relative to each other. We demonstrated a dual MLA based EPE that produced excellent exit pupil uniformity and better than $90 \%$ diffraction efficiency for all three wavelengths in a color display system. Registration was performed with sub-micron precision using farfield alignment techniques. Both numerical and experimental results are presented, and three fabrication technologies: grayscale lithography, photoresist reflow, and isotropic etching, are compared.
\end{abstract}

Keywords: microlens array, scanning, NA expander, micro-optics, displays

\section{INTRODUCTION}

Retinal Scanning Display (RSD) systems operate by scanning a light beam onto the viewer's retina in a 2-D raster format. ${ }^{1}$ RSD typically creates a small exit pupil that is about the size of the eye-pupil. To obtain a large exit pupil, an Exit Pupil Expander (EPE) (i.e., numerical aperture (NA) expander) needs to be placed at an intermediate image plane in the display system. Exit pupil expansion can be achieved using 2-D binary gratings, multi-level diffraction gratings, single microlens arrays, 2-D holographic gratings, fiber optic faceplates, and other diffuser approaches. However, none of these approaches produce a high degree of uniformity while maintaining high efficiency in multi-color displays.

In this paper we discuss a novel solution to the color exit pupil expansion problem using two identical microlens arrays that are precisely registered relative to each other and separated by one focal length distance. The EPE application in display systems provides a novel use for microlens arrays. In section 2, we discuss RSD optics and EPE operation. In section 3, we discuss the principle of operation of the dual microlens array concept, and present numerical and experimental results. We compare dual MLA performance with other microoptical elements and also compare performance of several micro-optics mastering and replication technologies we tested during this research.

\section{EXIT PUPIL EXPANDER OPERATION}

Figure 1 illustrates RSD optics and the EPE operation. ${ }^{2,3}$ The EPE is placed at an intermediate image plane between the scanners and the display exit pupil. A focused spot scans across the EPE and produces multiple diffraction orders at the exit pupil, where each diffraction order contains the full image information. The eye pupil samples a few of such diffraction orders and forms a retinal image. Overall luminance and color uniformity across the exit pupil perceived by the viewer is a function of the uniformity of the diffraction order relative intensities, focused spot size, microlens diameter, scanning beam profile, and the viewer's eye-pupil size.,

In typical RSDs, exit pupil size without an EPE is in the order of 1 to $3 \mathrm{~mm}$. To enlarge the numerical aperture (NA) of the incoming beam for the required exit pupil size, the EPE is placed at the intermediate image plane between the scanner and the exit pupil. Note that the Optical Invariant before and after the EPE plane does not remain constant in the presence of an EPE. A number of technologies such as controlled angle diffusers that are used for screens appear to be good EPE candidates. However, to reduce system size while maintaining resolution, the display pixel size $(p)$ and the size of the focused scanned spot incident on the EPE are designed to be smaller than $20 \mu \mathrm{m}$. The small spot size 
prohibits the use of diffuser materials, as they are dependent upon the illuminating beam being much larger than the features of the diffuser material.

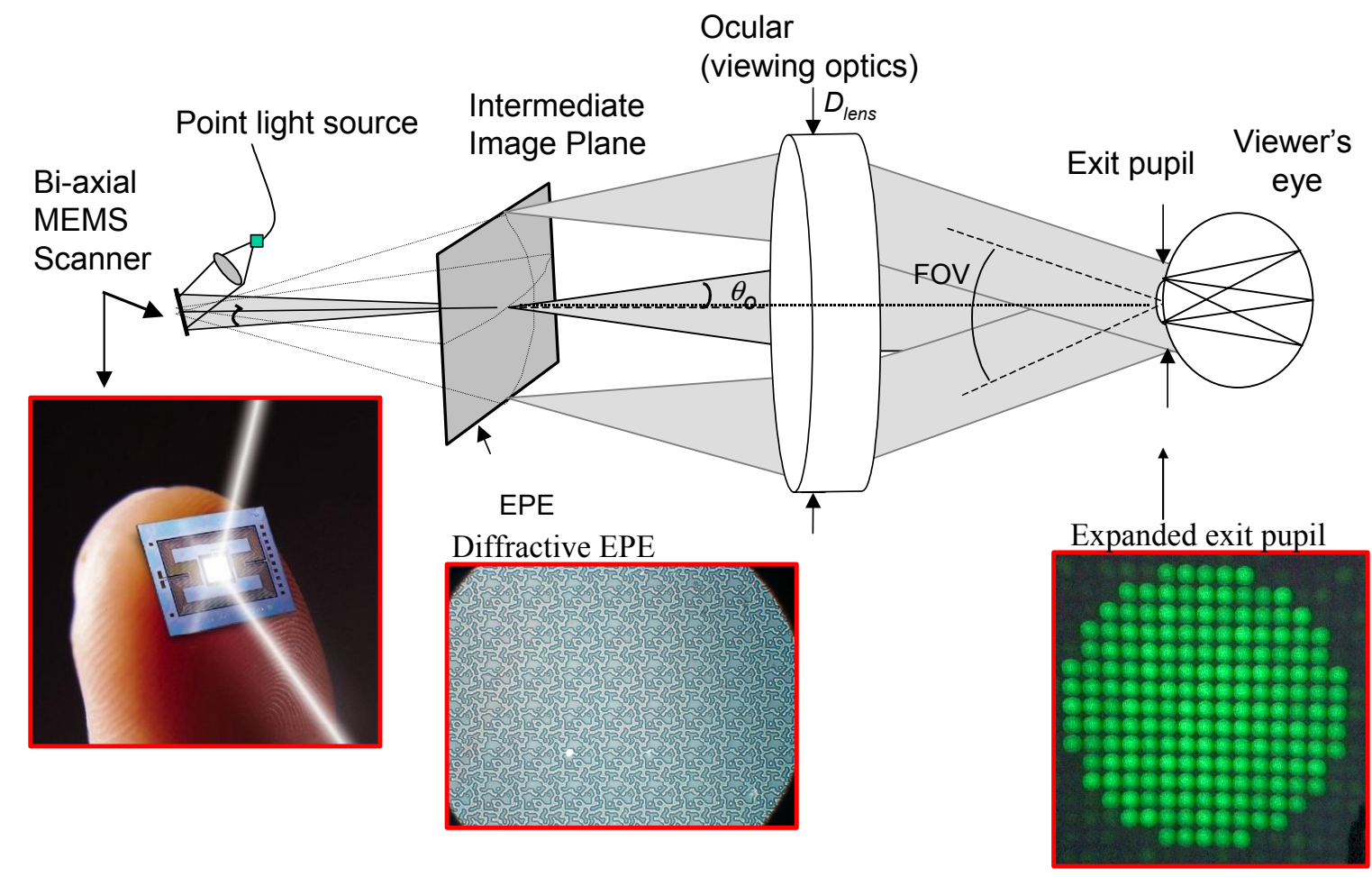

Figure 1: Scanned beam display optics including exit pupil expander (EPE) at the intermediate image plane. Expanded exit pupil is made up of an array of diffraction orders due to periodic structure of the 2-D diffraction grating illustrated in the figure.

Diffractive optical elements can be used to solve the NA expansion problem in monochrome displays. EPE efficiency is defined as the fraction of incident power transferred to the useful NA of the EPE. Diffraction efficiencies on the order of $65 \%$, which is within $2-3 \%$ of the theoretical limit, have been achieved in red monochrome and green monochrome displays. EPE uniformity is defined as the brightness variation within the useful NA of the EPE. Diffraction order uniformities better than $20 \%$ have been achieved in monochrome units. ${ }^{3}$ Monochrome DOEs have been incorporated into wearable display products by Microvision, Inc. DOEs work very well at the design wavelength but the intensity of the central $0^{\text {th }}$ order grows rapidly if the wavelength changes or if the etch depth of the grating does not meet the design specifications due to manufacturing tolerances. Figure 2 illustrates another problem with diffractive EPEs - the number of diffraction orders produced at each wavelength is the same but the diffraction angle for each order is proportional to the input wavelength. DOE performance versus color is also illustrated in Figure 4(a). If the display useful NA is defined as the overlapping 'white' exit pupil formed by the smallest wavelength in the system, typically blue, then the theoretical efficiencies of green and red reduces from about $70 \%$ to $50 \%$ and $35 \%$, respectively, which are significant losses in light power.

Even orders missing (EOM) binary $\mathrm{DOEs}^{5}$ can be used to reduce the bright central diffraction order problem in microdisplays, but the scaling of NA with wavelength remains to be an issue, limiting the efficiency. Likewise, multilevel DOEs can be used to achieve reduced $0^{\text {th }}$ order and better uniformity than binary gratings. Analysis showed that, an 8-level (3-mask) DOE is needed in order to achieve good exit pupil uniformity. Exit pupil profile corresponding to an 8level DOE is shown in Figure 2(b). A 3-level (2-mask) DOE is designed and fabricated to balance the central $0^{\text {th }}$ order for red, green, and blue laser illumination. Figure 2(a) illustrates a SEM picture of the 3-level DOE. In theory, a 3-level mask should do fairly well in balancing the $0^{\text {th }}$ order. However, as illustrated in Figure 2(c), alignment tolerances 
between different level masks even for a 2-level mask precludes achieving acceptable uniformity and efficiency when unit cell size is in the order of $20 \mu \mathrm{m}$ and output NA is in the order of 0.25 .

What makes color exit pupil expansion a difficult problem can be summarized as follows:

- High efficiency and good uniformity are both desired at the exit pupil plane

- Use of DOEs is ruled out due to poor efficiency and uniformity in the presence of multiple wavelengths

- EPE Surface should be cosmetically defect free as it is placed at an intermediate image plane

- The unit cell size of the periodic structure is small $(10-20 \mu \mathrm{m})$ and NA required after the EPE is large $(0.15$ 0.25 ), which pushes the limits of microoptics fabrication technology

\section{3-level DOE - SEM}

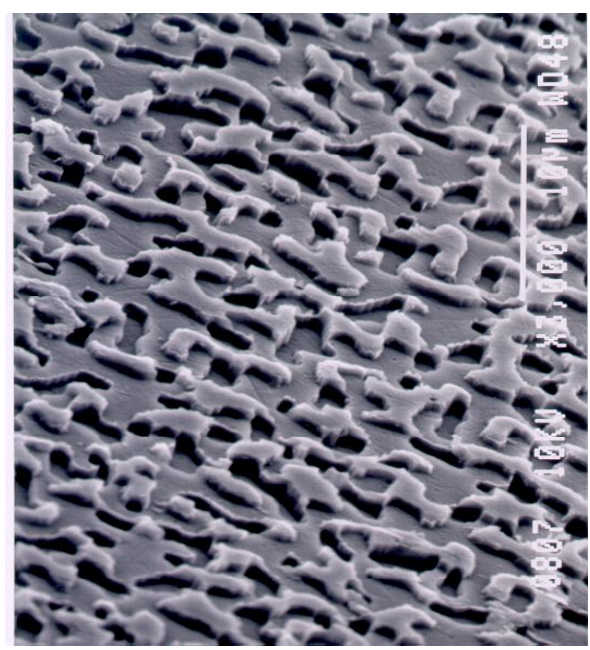

(a)

\section{Theoretical \\ Farfield Pattern}

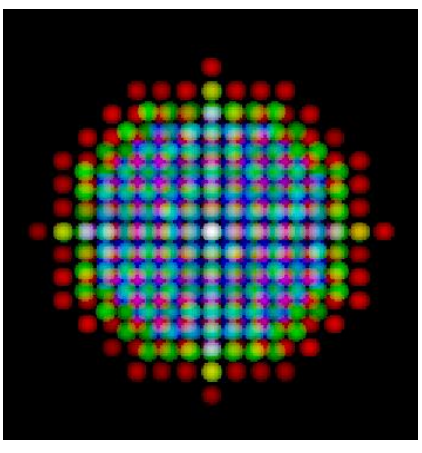

(b)
Actual

Farfield Pattern

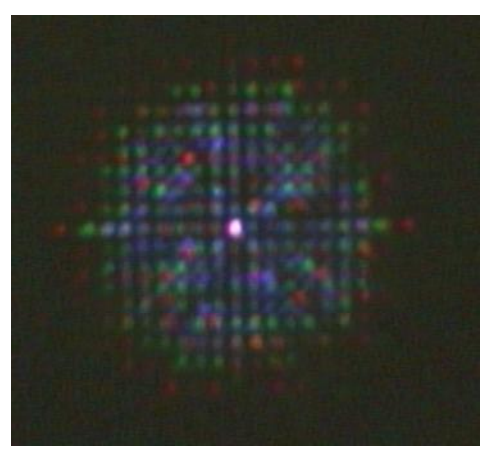

(c)

Figure 2: (a) 3-level (2-mask) DOE SEM picture; (b) Best theoretical color exit pupil pattern possible with an 8-level (3mask) DOE if all fabrication and mask alignment issues are resolved (notice scaling of exit pupil size with each wavelength; area of red circle is about twice that of blue circle); (c) Experimental exit pupil pattern produced by multi-level DOE in (a) shows very poor efficiency and color uniformity.

\section{DUAL MICROLENS ARRAY OPERATION}

A single microlens array functions in a way similar to DOEs and expands the NA of the beam. A novel approach to exit pupil expansion is the use of a dual microlens array (DMLA), where two identical microlens arrays (MLA) are cascaded with one focal length $(f)$ distance in between. Figure 3 compares NA expansion using one microlens array and the DMLA. Figures 3(b) and 3(e) illustrate beam propagation along the optical axis when a focussed Gaussian spot illuminates the microlens array. The simulation results are obtained using physical optics beam propagation using the Fresnel diffraction integral. ${ }^{6,7}$ The Gaussian spot covers one microlens fully and two microlenses partially and forms an exit pupil pattern in the farfield, which consists of a 2-D array of diffraction orders. Notice that both single MLA and dual MLA form a focused spot at the array focal plane then expand the beam out to the NA defined by the $f_{\#}=f / D$ of the MLA.

Each microlens in the single MLA configuration takes a segment of the incoming beam and forms its optical Fourier Transform, except for a quadratic phase factor, at the focal plane of each microlens. Free space propagation afterwards forms the expanded exit pupil in the farfield. Adding the second MLA adds the missing quadratic phase factor and makes each microlens pair a perfect Fourier transformer. Analytical formulation of the dual MLA operation will be published elsewhere. ${ }^{8}$

There are two very important advantages to the DMLA approach, (1) As illustrated in Figure 4(a) and 4(b), for DOEs the expanded NA scales with wavelength, while the expanded NA for the DMLA is determined only by the $f_{\#}$ of the 
microlenses and is independent of the wavelength, thus allowing all light to contribute to the useful NA of the microlens.- this results in very high efficiency for each color. Diffraction orders are formed within the useful NA of the MLA due to the periodicity of microlenses by overlap interference of light emanating from neighboring discrete microlens locations. The peak intensities of the resultant diffraction orders are uniform within $15 \%$ for the case of Gaussian spot illumination. Thus, the DMLA approach provides improved efficiency and exit pupil uniformity compared to DOEs.

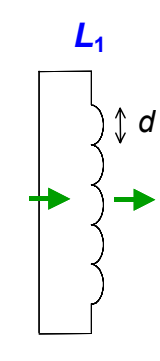

(a)

$L_{1} \quad L_{2}$

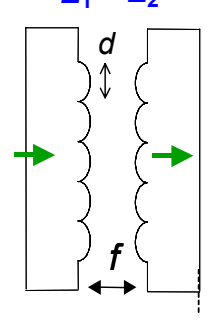

(d)

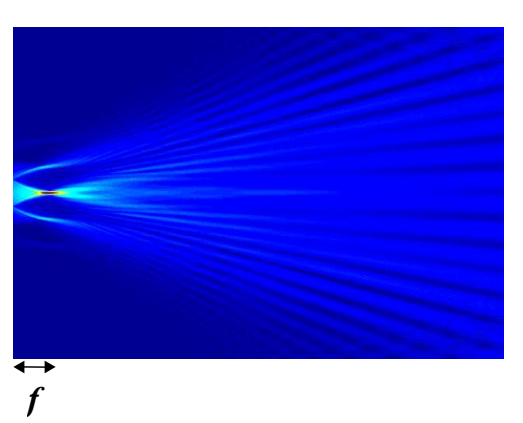

(b)

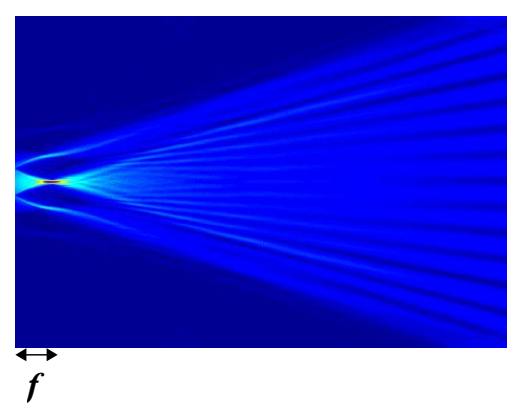

(e)

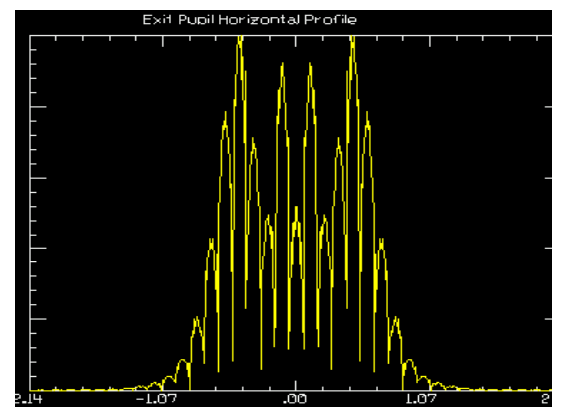

(c)

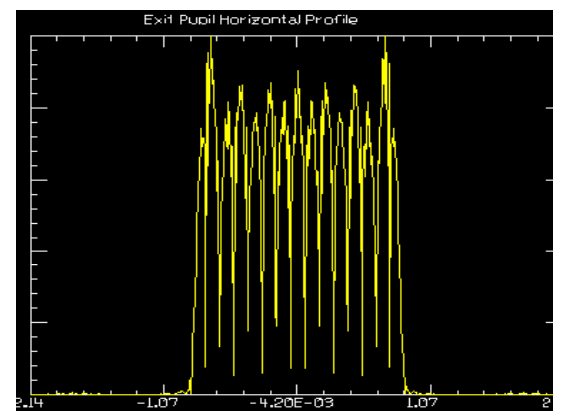

(f)

Figure 3 (a) Single microlens array profile; (b) physical optics beam propagation from microlens array patterned surface toward the farfield; (c) farfield exit pupil intensity cross-section; (d)-(e)-(f) same figures for the dual microlens array case.

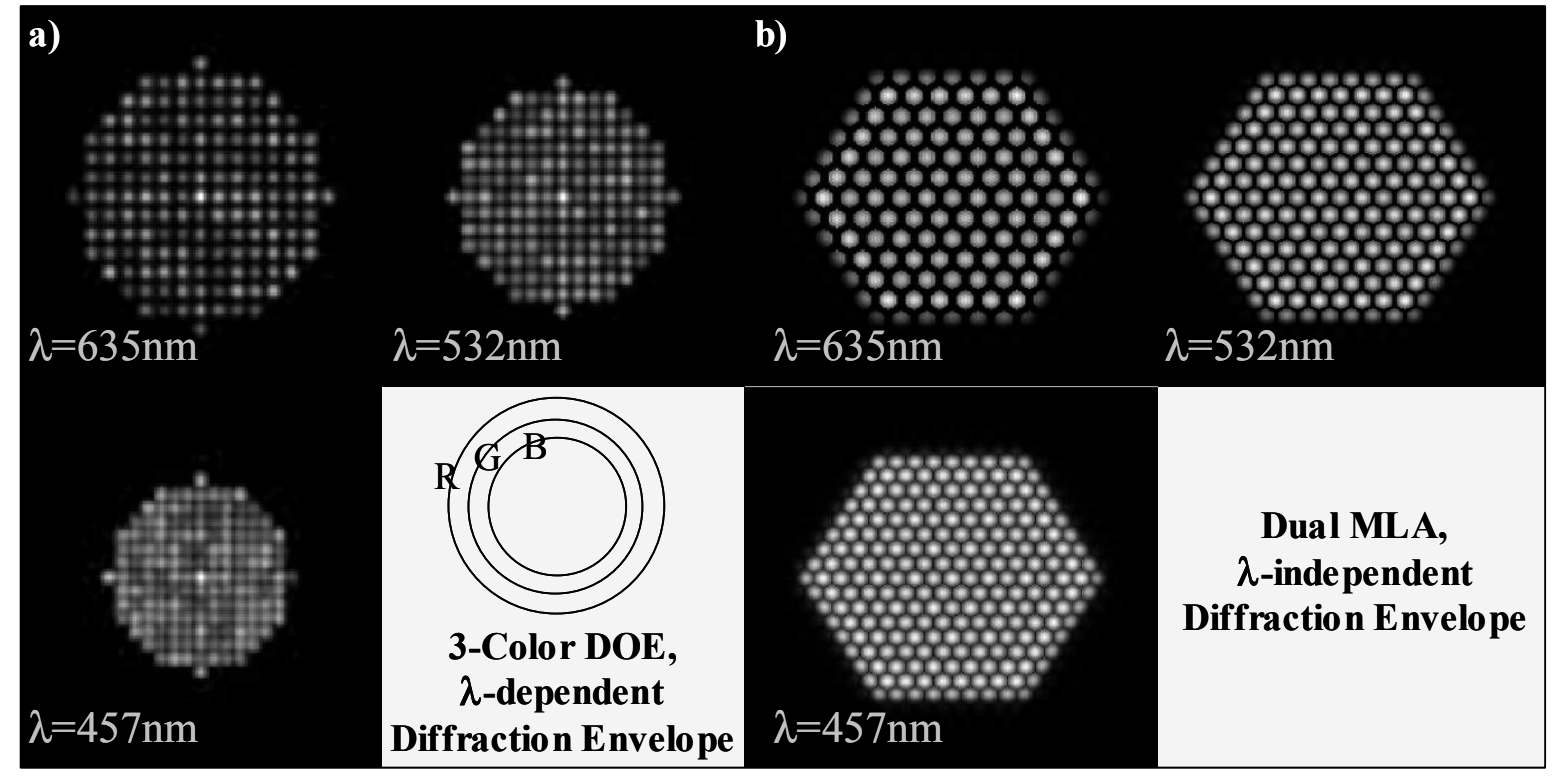

Figure 4. Comparison of diffraction envelope size versus wavelength for (a) 3-Color DOE and (b) Dual MLA EPE. 


\subsection{Alignment and Assembly of DMLA}

Figure 5 illustrates the computer-controlled setup designed to align and bond two identical microlenses with 6 degreesof-freedom: $\mathrm{x}, \mathrm{y}, \mathrm{z}$, tip, tilt, and rotation. Alignment tolerances are $\pm 0.5 \mu \mathrm{m}$ for in-plane registration $(\mathrm{x}, \mathrm{y}), \pm 1 \mu \mathrm{m}$ for MLA intermediate spacing (z), and better than \pm 3 mdeg for rotation, tip, and tilt. Alignment using near field techniques to such tight tolerances require sophisticated aligners with long-working-distance and high-magnification objectives. Even then, the alignment procedure using near field techniques would be difficult. The alignment procedure is simplified by observing the farfield diffraction pattern, which is the expanded exit pupil pattern. Different types of alignment errors produce interesting Moire-like artifacts when imaged. Using visual feedback from the farfield interference patterns and uniformity variations, one can correct all rotational and translational errors. The setup illustrated in the figure is not well suited for high-volume manufacturing, but it can be used for low and mid volume manufacturing. UV curing epoxy is placed in between the MLA and once the alignment procedure is completed, MLAs are fixed in place by UV exposure. The alignment and bonding of two microlens arrays using UV curing epoxy with required precision can be achieved within minutes.

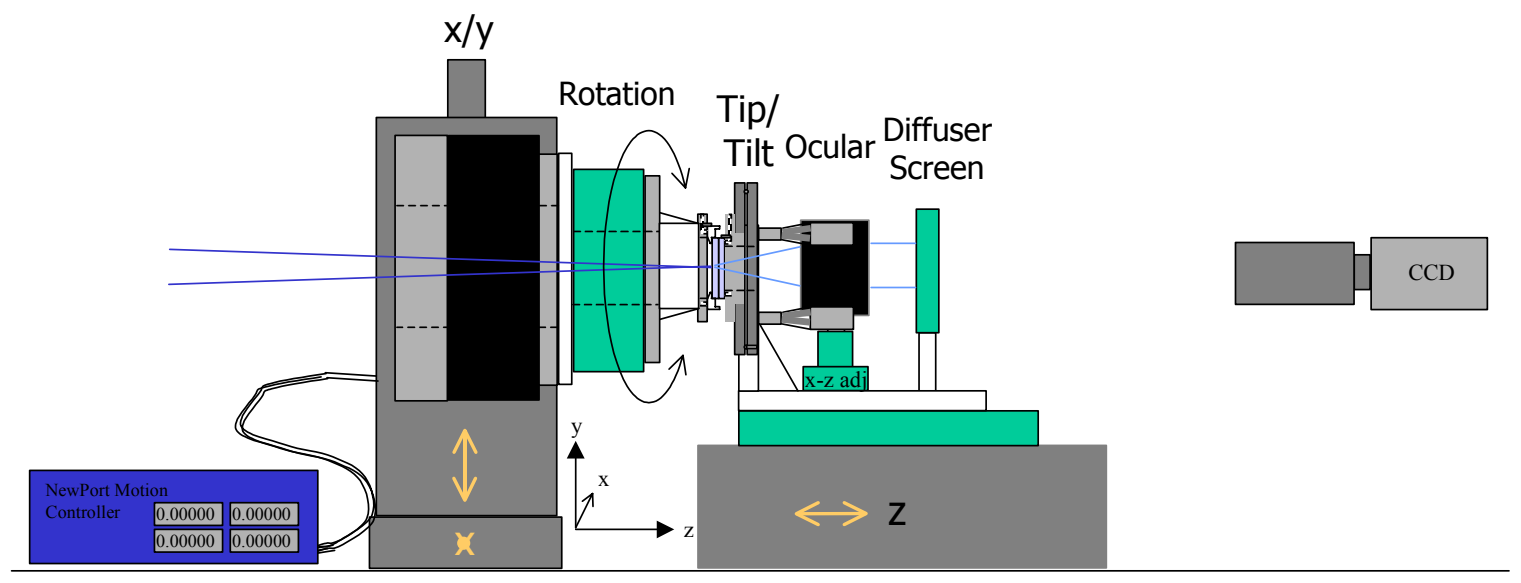

Figure 5 Dual microlens array alignment and bonding setup using far-field alignment techniques.

\subsection{Fabrication Technologies}

There are a number of fabrication technologies for making microlenses for a variety of applications. ${ }^{9}$ In our design, microlens diameter is $15 \mu \mathrm{m}$ and focal length is $30 \mu \mathrm{m}$. Once a good master is obtained, injection molding, stamping, and other techniques can be used to replicate the microlens arrays. There are always limitations to fabrication techniques determined by process types and inherent capability. In the process of implementing a usable Dual MLA Color EPE, a handful of issues were encountered which had to be overcome. In this paper, we discuss three of the principal issues, primarily related to obtaining good far-field uniformity from the EPE element. They involve seam sharpness or seam radius encountered in grayscale lithography, flat gap in the seam-areas encountered in photoresist reflow method, and flat-top profile encountered in isotropic etching subtractive process.

\section{Grayscale Lithography:}

Figure 6(a) illustrates a rectangular-packed perfect spherical microlens and Figure 6(b) illustrates the AFM surface profile measurement of a single microlens from an array produced using grayscale lithography. Grayscale lithography can involve imaging a grayscale mask through a projection aligner or direct exposure through contact printing, or can be achieved using laser writing on a substrate with analog photoresist. ${ }^{10,11}$ By adjusting the laser exposure duration or intensity, photoresist is hardened in varying amounts by spatially adjusting the exposure time and intensity, and after development and rinsing, 3-dimensional surface relief features with varying depth profile can be produced on the photoresist. Reactive ion etching (RIE) of the photoresist transfers the grayscale pattern onto the glass substrate underneath the resist. As illustrated in Figure 6(b), edges and corners of the microlenses are not sharp. Furthermore, step and repeat exposure of the substrate results in stitching errors on the surface, which are easily seen by the eye. 
The impact of smoothing of corners and edges on the exit pupil pattern can be accurately simulated using physical optics beam propagation. Our numerical results and the experimentally observed farfield patterns agree very well. Figure 7 illustrates the microlens profiles and the corresponding farfield patterns for 3 different values of seam radius. Based on the farfield uniformity requirement of $20 \%$, the maximum acceptable seam radius is determined to be $1.5 \mu \mathrm{m}$, which proved to be difficult to achieve.

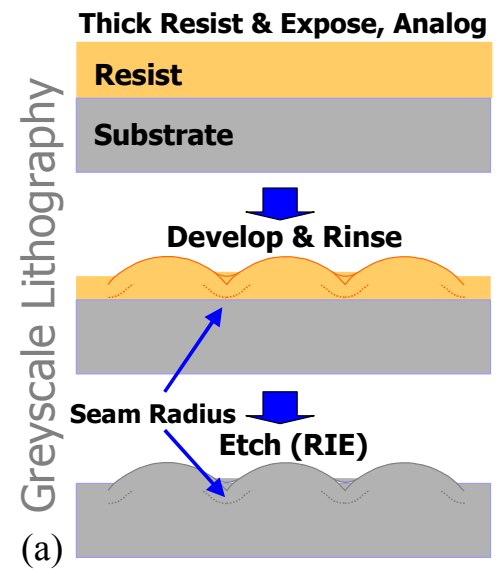

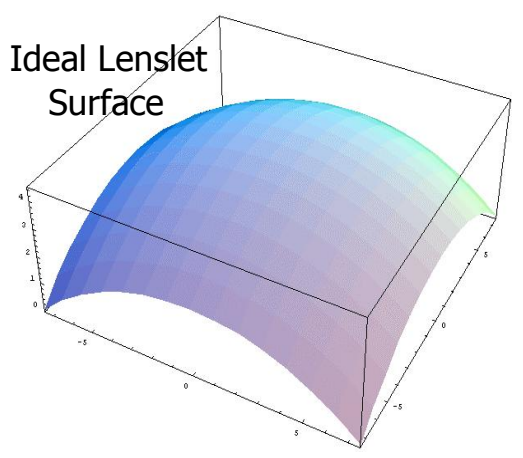

(b)

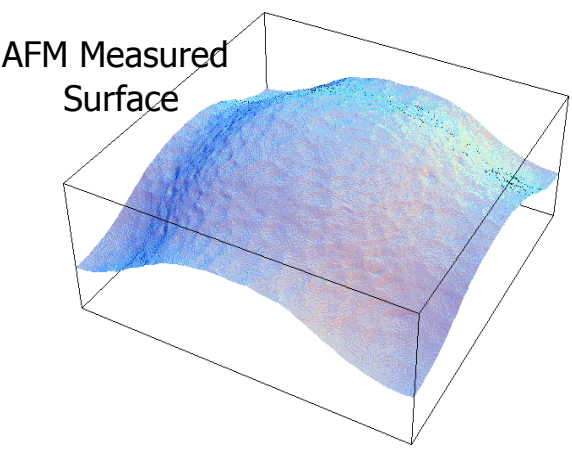

(c)

Figure 6: (a) microfabrication sequence for producing microlenses using grayscale lithography; (b) desired microlens profile; (c) actual microlens surface profile measured with AFM. Notice the rounding in the corners and the surface roughness.

\section{Photoresist Reflow:}

The photoresist reflow technique is a simple process where the master is made by patterning the photoresist then melting the photoresist pillars to form a hemispherical surface profile by surface tension. The resist volume controls the $f_{\#}$ of the microlenses. Microlenses of this type show excellent surface profile and surface quality, however, a small gap remains between the microlenses and the light that goes through the gap creates a hotspot at the center of the exit pupil, degrading the farfield uniformity. Figure 8 shows the microlens profiles and the exit pupil profiles as a function of gap spacing. The technology limit for the minimum gap is about $1 \mu \mathrm{m}$, which yields an even larger gap at the corners. Adding an absorptive masking material in the gap areas can block the light from exiting through the gap and make the uniformity better than any other technology, but the masking reduces the efficiency to about $50 \%$ for an approximate $2 \mu \mathrm{m}$ gap. Addition of the absorptive layer also complicates the process by adding one more lithography step. Exit pupil patterns produced by photoresist reflow MLAs with masking of the gap areas is illustrated in Figure 8 (bottom row).

\section{Isotropic-Etched Subtractive Process:}

A substrate is etched using isotropic etching through an array of pinholes and forms the master mold. Microlens arrays can then be produced on plastic using a number of replication technologies. Microlens profile is determined by etch selectivity in horizontal and vertical directions. The size of the etch hole results in a flattop at the center of each microlens. Figure 9 illustrates the effect of the etch-hole diameter on the exit pupil uniformity. Once we achieved flattop sizes smaller than $1.5 \mu \mathrm{m}(\sim 10 \%$ of the microlens diameter), the efficiency of DMLA produced with this approach is measured to be better than $90 \%$ for all colors and uniformity is better than $20 \%$. The excellent farfield performance, excellent surface quality, and suitability for high-volume manufacturing offered by this approach make it very attractive for making MLAs.

To give an indication of the full array size, a bonded Dual MLA is shown in Figure 10. One has to keep in mind that there are greater than 2 million microlens sets in this array. The diffraction efficiency is on the order of $>90 \%$ into the desired exit pupil pattern. The particular arrays that were used to fabricate this EPE were replicated using UV casting, and can be fabricated in a fairly cost-effective manner. 
Grayscale Lithography

$15 \mu \mathrm{m}$ diameter microlenses
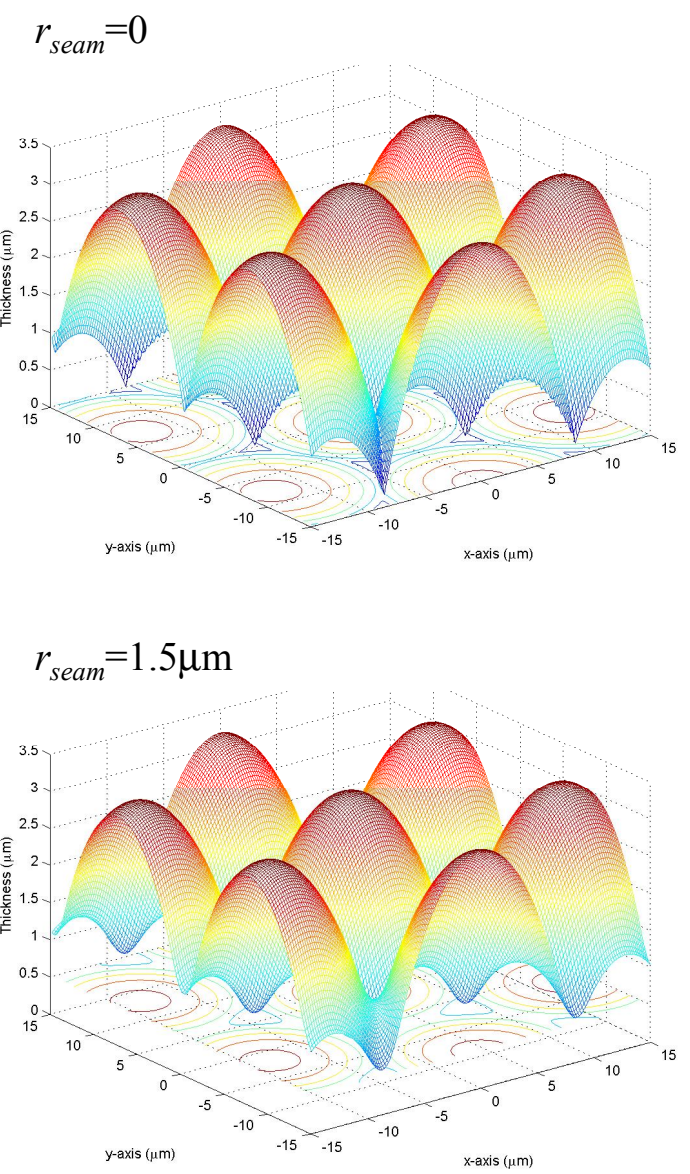

$$
r_{\text {seam }}=3 \mu \mathrm{m}
$$

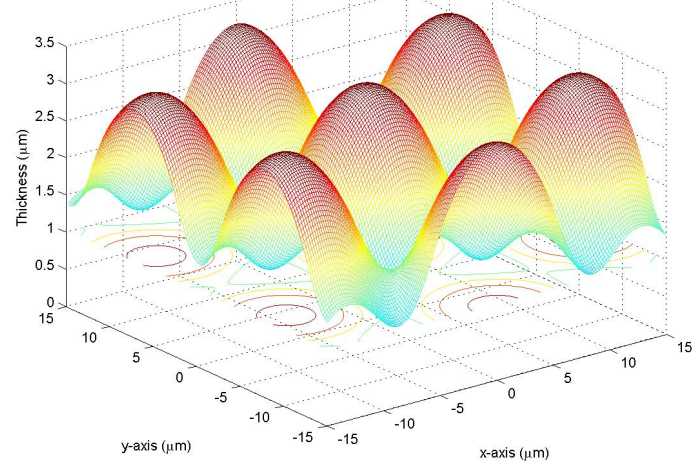

Farfield patterns
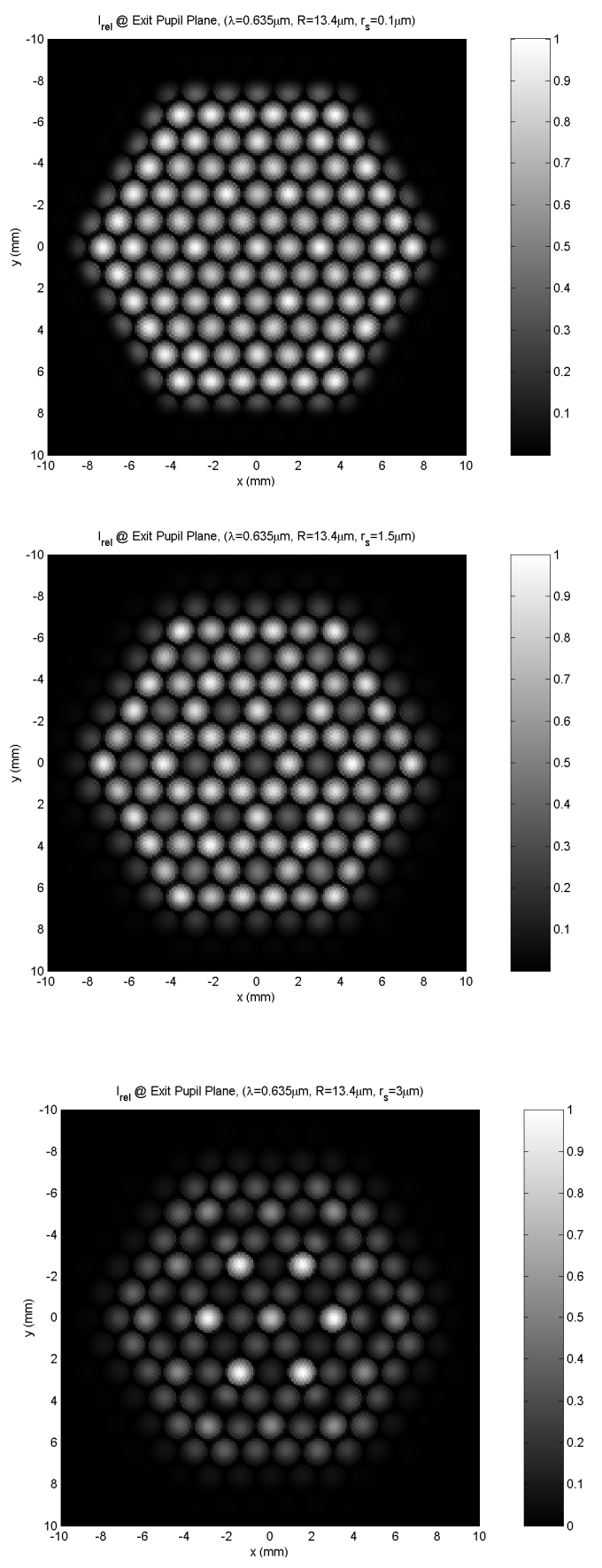

Figure 7: Simulated profiles for microlenses produced by grayscale lithography and the corresponding exit pupil patterns. Different rows show different seam radii of $0 \mu \mathrm{m}, 1.5 \mu \mathrm{m}$, and $3 \mu \mathrm{m}$. 


\section{Photoresist Reflow}

\section{$15 \mu \mathrm{m}$ diameter microlenses}

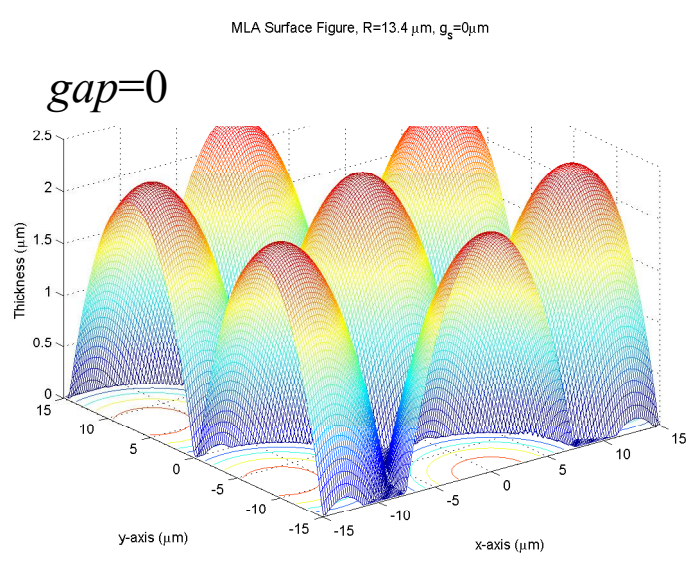

MLA Surface Figure, $R=13.4 \mu \mathrm{m}, g_{\mathrm{s}}=2.5 \mu \mathrm{m}$

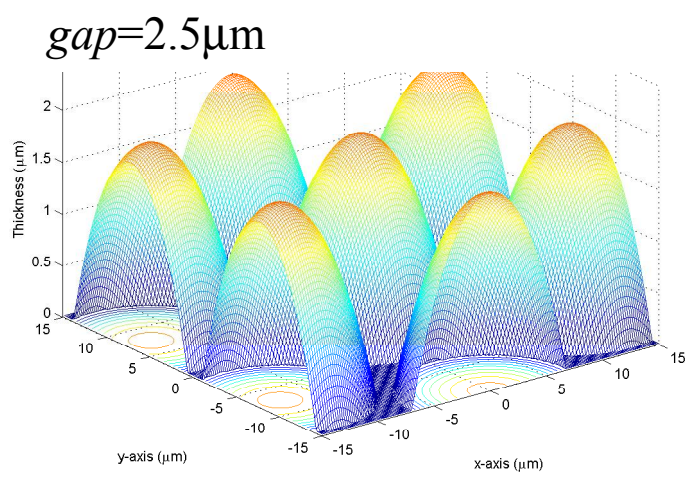

MLA Surface Figure, $R=13.4 \mu \mathrm{m}, \mathrm{g}_{\mathrm{s}}=2.5 \mu \mathrm{m}$

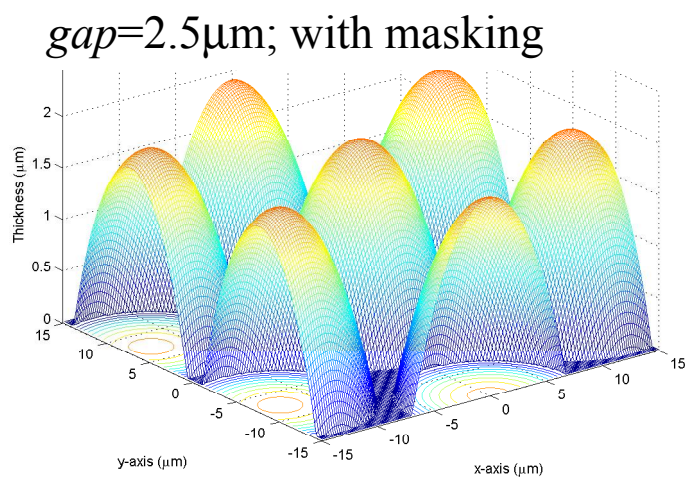

\section{Farfield patterns}
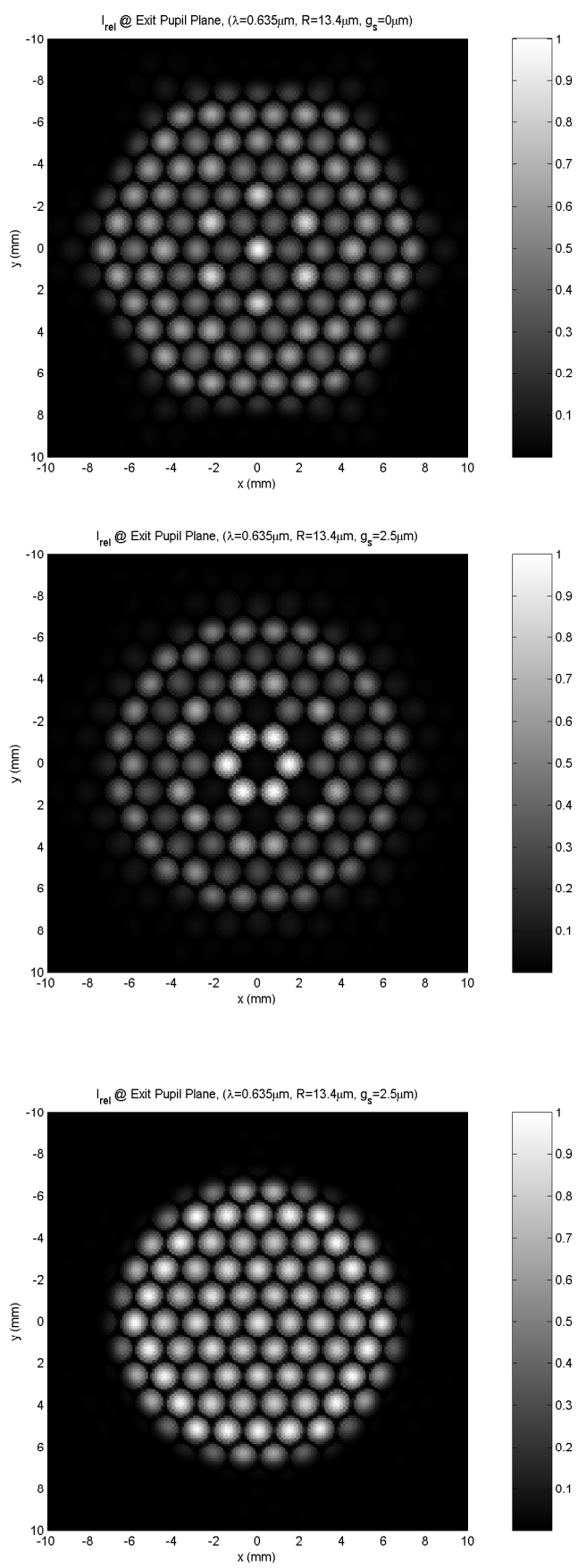

Figure 8: Simulated profiles for microlenses produced by photoresist reflow method and the corresponding exit pupil patterns. Top row: minimum gap of $0 \mu \mathrm{m}$ such that flat gap regions only exist in hexagonal corners, Middle row: minimum gap of $2.5 \mu \mathrm{m}$, and Bottom row: minimum gap of $2.5 \mu \mathrm{m}$ with opaque masking over flat region showing excellent performance in uniformity. 
Isotropic Etching Process

$15 \mu \mathrm{m}$ diameter microlenses

\section{Flattop width $=0$}

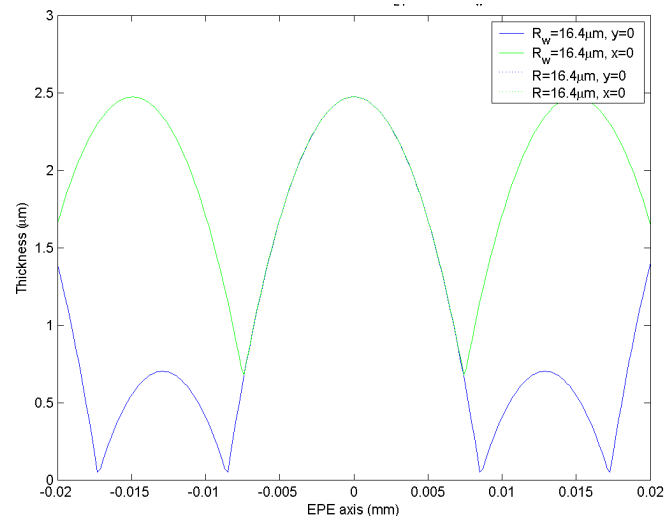

\section{Flattop width $=1.5 \mu \mathrm{m}$}

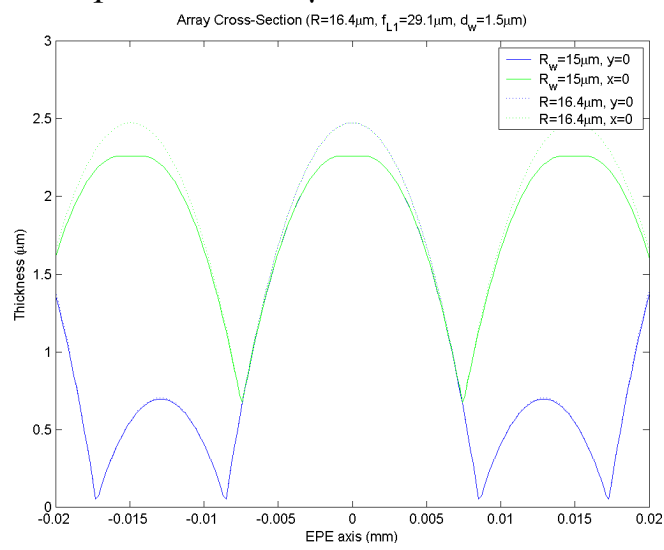

Flattop width $=2.5 \mu \mathrm{m}$

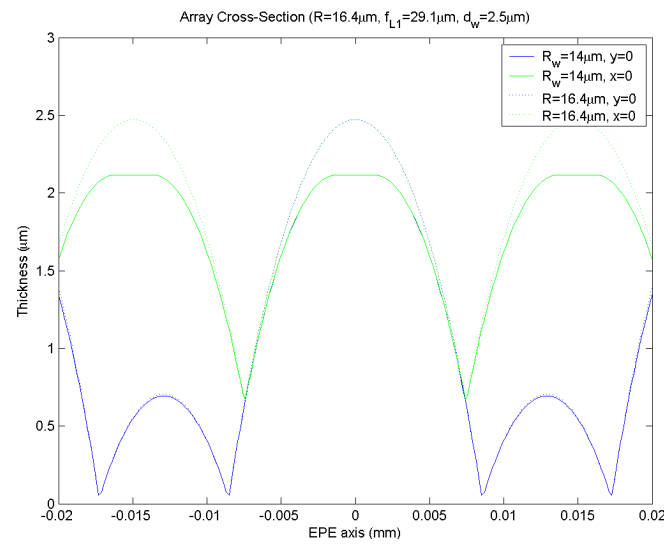

Farfield patterns
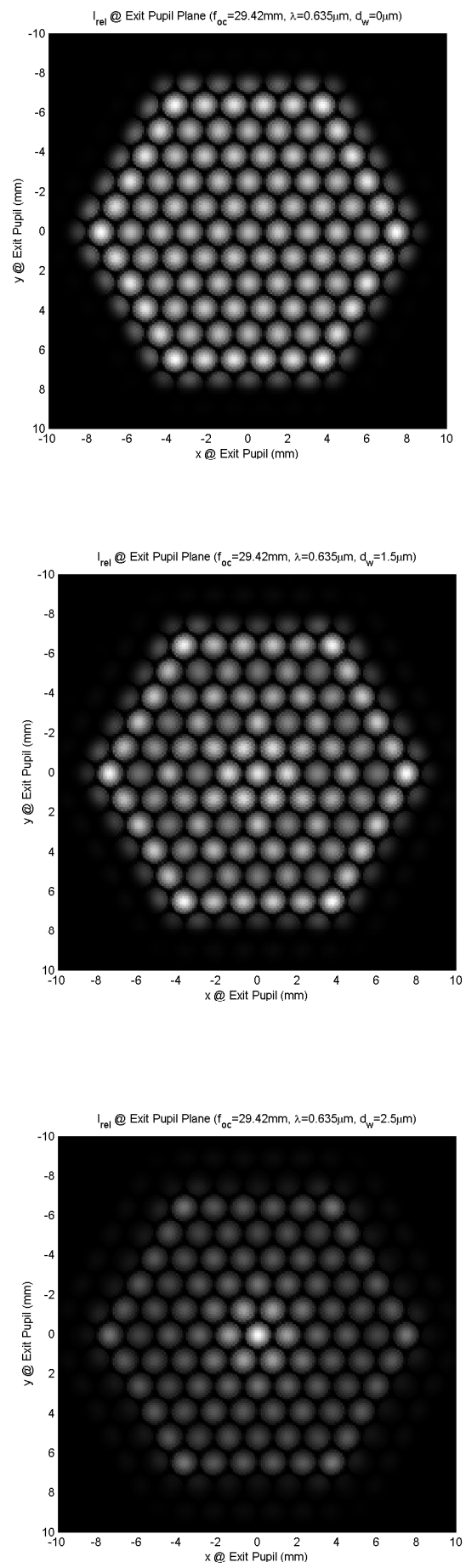

Figure 9: Simulated profiles for microlenses produced by an isotropic etching method and the corresponding exit pupil patterns. Different rows show different flat-top widths of $0 \mu \mathrm{m}, 1.5 \mu \mathrm{m}$, and $2.5 \mu \mathrm{m}$. 


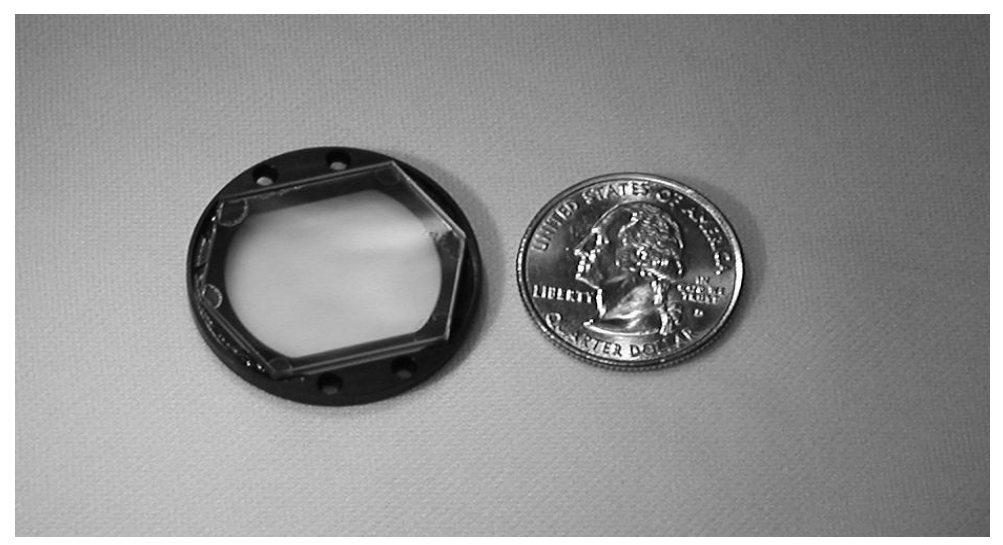

Figure 10: Picture of Dual MLA Color EPE, yielding uniformity better than $20 \%$ and efficiency better than $90 \%$ for all colors.

\section{CONCLUSIONS}

Dual MLA is a novel approach that solves the difficult problem of exit pupil expansion in full color displays. Many other technologies such as various types of diffraction gratings and holographic approaches were tried and dual MLA approach proved to be the best approach. We also investigated a number of different fabrication technologies for making the MLAs. Each approach has an associated artifact, which is illustrated by experiments and numerical simulations. The technology best suited for our requirements proved to be the isotropic-etched subtractive process for master making, the replication can be done using a number of plastic replication technologies. Using the DMLA approach, the resultant efficiency for red, green, and blue are all $>90 \%$ and the uniformity is better than $20 \%$ across the display exit pupil. A challenge we could present to microlens makers is to develop a replication technology that allows stamping microlenses with submicron registration tolerances from both sides on thin substrates $(50-100 \mu \mathrm{m}$ thick). We can then produce the DMLA on a single substrate without requiring separate alignment and bonding.

\section{ACKNOWLEDGMENTS}

We are grateful to Peggy Lopez (Microvision Inc.) for her help with the experimental work and the whole HighPerformance Products Team at Microvision for their support.

\section{REFERENCES}

1. H. Urey, Retinal Scanning Displays, in Encyclopedia of Optical Engineering, R. Driggers, Editor, Vol. 3, p. 24452457, Marcel Dekker, 2003.

2. H. Urey, "Spot size, depth of focus, and diffraction ring intensity formulas for truncated Gaussian beams," Applied Optics, Vol. 43, No. 3, p. 620-625, Jan 2004

3. H. Urey, "Diffractive Exit-Pupil Expander for Display Applications," Appl. Opt. 40, 5840-5851 (2001)

4. K. Powell, H. Urey, "A Novel Approach to Exit Pupil Expansion for Wearable Displays", Proc. SPIE, vol. 4711, pp.235-248 (2002)

5. H. Urey, United States Patent No: 6,639,719, Oct. 2003

6. M. Brown, M. Bowers, "High Energy, Near Diffraction Limited Output from Optical Parametric Oscillators Using Unstable Resonators," Proc. SPIE, vol. 2986, pp.17 (1997).

7. J. W. Goodman, Introduction to Fourier Optics, 2nd Ed., John Wiley and Sons, 1994

8. H. Urey and K. D. Powell, "Color exit pupil expander using microlens arrays" in preparation for Applied Optics

9. H. Sankur and E. Motamedi, "Microoptics development in the past decade," in Micromachining Technology for Micro-Optics, Proc. SPIE, Vol. 4179, pp. 30-55

10. M. T. Gale, M. Rossi, J. Petersen, and H. Schutz, "Fabrication of continuous-relief micro-optical elements by direct writing in photoresist," Opt. Eng., Vol. 33, pp. 3556-3566, 1994.

11. T. Hessler, Continuous-Relief Diffractive Optical Elements: Design, Fabrication, and Applications, Univ. Neuchatel, PhD Thesis, 1998 